\title{
Robust linear optimization under new distance measure
}

\author{
Zhang Jianke ${ }^{1,2 \star}$, Liu Sanyang ${ }^{1}$ and Ma Xiaojue ${ }^{1,2}$ \\ ${ }^{1}$ Department of Science, Xidian University, Xi'an 710071, China. \\ ${ }^{2}$ Department of Applied Mathematics and Physics, Xi'an University of Posts and Telecommunications, Xi'an 710121, \\ China.
}

Accepted 20 January, 2012

\begin{abstract}
Several robust counterparts of linear optimization problems with uncertain data were proposed since 1970 and have been extensively studied and extended. In these approaches, the uncertainty set plays an important role since it determines the level of protection of the solution; the solution might be too conservative in order to ensure that the solution remains feasible if the disturbance of data is relatively large. In this paper, we propose a new robust counterpart under a new distance measure. The new approach can ensure that all uncertain data can be mapped to a bounded neighborhood of nominal value regardless of the data from the nominal value either near or far. So the new approach succeeds in reducing the price of robustness; on the other hand, the new robust formulation is also a linear optimization problem. Numerical results for the problems of AFIRO and ADLITTLE from the Net Lib library shown that the effectiveness of the new formulation.
\end{abstract}

Key words: Linear programming, data uncertainty, robust linear optimization.

\section{INTRODUCTION}

Nowadays, robust optimization is widely used to optimize real world problems, such as the study by Ben-Tal and Nemirovski $(1999,2000)$ on linear optimization which showed that, in real world applications of linear programming, one cannot ignore the possibility that a small uncertainty in the data can make the usual optimal solution completely meaningless from a practical viewpoint. So the major difficulty we are faced with is how to seek a robust solution, which is immunized against the effect of data uncertainty. The robustness of solution is that the ability of a solution remain feasible with respect to data changes.

The earliest date of studies on robust optimization can be dated back to 1973 (Soyster, 1973). Soyster (1973) proposed the first robust model for linear optimization problems with uncertain data. However, the model is very conservative in the sense that they protect against the worst-case scenario. The interest in robust formulations in the optimization community was revived in the 1990s. A number of important robust formulations and

\footnotetext{
"Corresponding author. E-mail: jiankezh@163.com.
}

applications were introduced by Ben-Tal and Nemirovski (1999, 2000), Ghaoui and Lebret (1997) and Ghaoui et al. (1998). They provided a detailed analysis of the robust optimization framework in linear optimization and general convex programming. However, as the resulting robust formulations involve conic quadratic problems, Bertsimas and $\operatorname{Sim}(2004,2006)$ proposed a different approach to control the level of conservatism in the solution that has the advantage which leads to a linear optimization model. Bertsimas et al. (2004) also researched the robust counterpart of linear optimization with uncertainty set described by an arbitrary norm. Ben-Tal et al. (2004) considered linear programs with uncertain parameters. In these problems, part of the variables must be determined before the realization of the uncertain parameters ("nonadjustable variables"), while the other part are variables that can be chosen after the realization ("adjustable variables"). They introduced the adjustable robust counterpart (ARC) to this situation. Chen and Zhang (2009) extended affinely ARC to modeling and solving multistage uncertain linear programs with fixed recourse. Bertsimas and Brown (2009) used the theory of coherent risk measures to propose a methodology for constructing uncertainty sets within the framework of robust 
optimization for linear optimization problems with uncertain parameters. Recently, the methodology of robust optimization has been applied to a lot of practical problems.

Despite its tractability, one of the main criticisms of these robust optimization formulations is that these methods may have lost too much optimality to guarantee the robustness of the solution. In some practical problems, the robust solution may not be meaningful for decision-making if it is too conservative. Thus, the present number of studies is trying to give new models, which make the solution to ensure the robustness, but its optimality will not lose a lot.

In this paper, we propose a new counterpart for linear optimization with uncertain data. Firstly, by analyzing, we can see that the construction of Soyster (1973) model is based on Euclidean distance. Because of this, when the difference between the real data and the nominal data is relatively large, the feasible region of this model will become relatively small, which led the model to be too conservative, and the robust solution will have too much of loss of optimality. In order to reduce the conservatism of this model, we introduce a new distance metric (Wu and Yang, 2002; Zhang and Chen, 2004) to research the problems of c-means clustering and fuzzy c-means (AFCM) clustering. The new distance metric can be sure that all uncertain data can be mapped to a bounded neighborhood of nominal value regardless of the data from the nominal value either near or far. Thus, we use it to create a new robust optimization counterpart. The new approach succeeds in reducing the price of robustness. On the other hand, the new robust formulation is also a linear optimization problem.

\section{ROBUST COUNTERPART OF LINEAR PROGRAMMING PROBLEMS}

We consider the nominal linear optimization problem as following:

$$
\begin{array}{ll}
\max & c^{T} x \\
\text { s.t. } & A x \leq b \\
& l \leq x \leq u
\end{array}
$$

In the Equation 1, we assume that data uncertainty only affects the elements in matrix $A$. Without loss of generality; we assume that the objective function $c$ is not uncertainty. Since we can use the objective maximize $z$, add the constraint $z-c^{T} x \leq 0$, and include this constraint into $A x \leq b$. Where $[A, b, c, l, u] \in Z, x \in \mathbf{R}^{n}$ and $Z \subset \mathbf{R}^{m \times n} \times \mathbf{R}^{m} \times \mathbf{R}^{n} \times \mathbf{R}^{n} \times \mathbf{R}^{n}$.

Consider a particular row $i$ of the matrix $A$ and let $J_{i}$ represent the set of coefficients in row $i$ that are subject to uncertainty (BenTal and Nemirovski, 2000; Bertsimas and Sim, 2004). Each data $a_{i j}, j \in J_{i}$ is modeled as asymmetric and bounded random variable $\tilde{a}_{i j}, j \in J_{i}$ that takes values in $\left[a_{i j}-\widehat{a}_{i j}, a_{i j}+\widehat{a}_{i j}\right]$ .variable $\tilde{a}_{i j}, j \in J_{i}$ that takes values in $\left[a_{i j}-\widehat{a}_{i j}, a_{i j}+\widehat{a}_{i j}\right]$. Associated with the uncertain data $\tilde{a}_{i j}$, we define the random variable $\eta_{i j}=\left(\tilde{a}_{i j}-a_{i j}\right) / \hat{a}_{i j}$, which obeys an unknown but symmetric distribution and takes values in $[-1,1]$.

\section{Robust counterpart of Soyster (1973)}

Soyster (1973) established the first robust counterpart for linear optimization with uncertain data. He considered each column $\tilde{A}_{j}$ of the realization matrix $\tilde{A}$ that belongs to a convex set. He showed that the problem is equivalent to a problem with linear constraints as follows:

$\max \quad c^{T} x$

s.t. $\quad \sum_{j} a_{i j} x_{j}+\sum_{j \in J_{i}} \hat{a}_{i j} y_{j} \leq b_{i} \quad \forall i$

$$
\begin{aligned}
& -y_{j} \leq x_{j} \leq y_{j} \quad \forall j \\
& l \leq x \leq u \\
& y \geq 0
\end{aligned}
$$

Let $X^{*}$ be the optimal solution of Equation 2, clearly, $y_{j}=\left|x_{j}^{*}\right|$, and thus

$\sum_{j} \tilde{a}_{i j} x_{j}^{*}=\sum_{j} a_{i j} x_{j}^{*}+\sum_{j \in J_{i}} \hat{a}_{i j} x_{j}^{*} \leq \sum_{j} a_{i j} x_{j}^{*}+\sum_{j \in J_{i}} \hat{a}_{i j}\left|x_{j}^{*}\right| \leq b_{i} \quad \forall i$ We can see that $\sum_{j \in J_{i}} \hat{a}_{i j}\left|x_{j}\right|$ gives the necessary "protection" of every constraint. Thus, the solution is robust.

\section{Robust counterpart of Ben-Tal and Nemirovski $(1999,2000)$}

The formulation of Soyster (1973) is the most conservative in practice although it admits the highest protection. So that the solution has an objective function value much worse than the objective function value of the solution of the nominal linear optimization problem. In order to reduce this conservatism, Ben-Tal and Nemirovski $(1999,2000)$ proposed the following robust counterpart.

$\max \quad c^{T} x$

$$
\begin{array}{ll}
\text { s.t. } & \sum_{j} a_{i j} x_{j}+\sum_{j \in J_{i}} \hat{a}_{i j} y_{i j}+\Omega_{i} \sqrt{\sum_{j \in J_{i}} \hat{a}_{i j}^{2} z^{2}} \leq b_{i j} \quad \forall i \\
& -y_{j} \leq x_{j}-z_{i j} \leq y_{j} \quad \forall i, j \in J_{i} \\
& l \leq x \leq u \\
& y \geq 0
\end{array}
$$


where $\Omega \geq 0$ is a positive parameter. It is called "safety parameter" and is used to adjust the robustness and optimality of the solution. The authors have shown that the probability of the $i$ constraint is violated at most $\exp \left(-\Omega^{2} / 2\right)$. The robust model of Equation 3 is less conservative than model of Equation 2, but model of Equation 3 is a second-order cone problem. Since model of Equation 3 is a nonlinear problem, a practical drawback of such a formulation is that it is more demanding computationally than the earlier linear models by Soyster (1973).

\section{Robust counterpart of Bertsimas and Sim (2004)}

Bertsimas and Sim (2004) proposed an approach that can decrease what we call the price of robustness. Especially, the attractive aspect of their method is that the formulation is also a linear optimization problem. This formulation can be extended to discrete optimization problems in a tractable way. The formulation is as follows:

$\max c^{T} x$

s.t.

$$
\sum_{j} a_{i j} x_{j}+\max _{\left\{S_{i} \cup\left\{t_{i}\right\}\left|S_{i} \subseteq J_{i},\right| S_{i} \mid=\left\lfloor\Gamma_{i}\right\rfloor t_{i} \in J_{i} / S_{i}\right\}}
$$$$
\left\{\sum_{j \in S_{i}} \hat{a}_{i j} y_{j}+\left(\Gamma_{i}-\left\lfloor\Gamma_{i}\right\rfloor\right) \hat{a}_{i t_{i}} y_{t}\right\} \leq b_{i}
$$

$-y_{j} \leq x_{j} \leq y_{j}$

$\forall j \in J_{i}$

$l \leq x \leq u$

$y \geq 0$

For every $i$, the parameter $\Gamma_{i}$ is not necessarily integer, that takes values in the interval $\left[0,\left|J_{i}\right|\right]$. The role of the parameter $\Gamma_{i}$ is to adjust the robustness of the method against the level of conservatism of the solution. If $\Gamma_{i}=\left|J_{i}\right|$, we have Soyster's method. Especially, model of Equation 4 has an equivalent linear formulation as follows:

$\max \quad c^{T} x$

s.t.

$$
\sum_{j} a_{i j} x_{j}+z_{i} \Gamma_{i}+\sum_{j \in J_{i}} p_{i j} \leq b_{i} \quad \forall i
$$

$$
\begin{array}{lc}
z_{i}+p_{i j} \geq \widehat{a}_{i j} y_{j} & \forall i, j \in J_{i} \\
-y_{j} \leq x_{j} \leq y_{j} & \forall j \\
l_{j} \leq x_{j} \leq u_{j} & \forall j \\
p_{i j} \geq 0 & \forall i, j \in J_{i} \\
y_{j} \geq 0 & \forall j \\
z_{i} \geq 0 & \forall i
\end{array}
$$

Bertsimas and Sim (2004) have shown that the probability of the $i$ constraint is violated at most $\exp \left(-\Gamma_{i}^{2} / 2\left|J_{i}\right|\right)$.

\section{THE NEW ROBUST COUNTERPART}

Here, we propose a new robust counterpart under new distance measure, that is, a linear problem is able to withstand parameter uncertainty under the model of data uncertainty $U$ without excessively affecting the objective function. Firstly, we propose a new distance measure based on analysis the Equation 2 model.

\section{The new distance measure}

The model of Soyster (1973) can be written as follows:

$$
\begin{array}{ll}
\max & c^{T} x \\
\text { s.t. } & \sum_{j} a_{i j} x_{j}+\sum_{j \in J_{i}} \hat{a}_{i j} y_{j} \leq b_{i} \quad \forall i \quad \quad \quad \longrightarrow \\
& -y_{j} \leq x_{j} \leq y_{j} \quad \forall j \\
& l \leq x \leq u \\
& y \geq 0 \\
\max & c^{T} x \\
\text { s.t. } \quad & \sum_{j} a_{i j} x_{j}+\sum_{j \in J_{i}} \operatorname{dist}\left(\tilde{a}_{i j}, a_{i j}\right) y_{j} \leq b_{i} \quad \forall i \\
& -y_{j} \leq x_{j} \leq y_{j} \quad \forall j \\
& l \leq x \leq u \\
& y \geq 0
\end{array}
$$

where $\operatorname{dist}\left(\tilde{a}_{i j}, a_{i j}\right)$ is $\operatorname{dist}\left(\tilde{a}_{i j}, a_{i j}\right)=\left|\tilde{a}_{i j}-a_{i j}\right|=\hat{a}_{i j}$. In other words, we understand this model from a broader sense; we can see that the necessary "protection" of every constraint is $\sum_{j \in J_{i}} \operatorname{dist}\left(\tilde{a}_{i j}, a_{i j}\right) y_{j}$. The $\operatorname{dist}\left(\tilde{a}_{i j}, a_{i j}\right)$ represents the maximum distance between the real value and nominal value.

Wu and Yang (2002) and Zhang and Chen (2004) proposed a new distance measure to research the problems of AFCM clustering. Suppose $x, y \in X$ and $X$ are compact subsets of $R^{n}$, let $\operatorname{dist}(x, y)$ denote the distance function between two vectors $x$ and $y$. Then the new distance will be as follows:

$\operatorname{dist}(x, y)=\sqrt{1-\exp \left(-\beta\|x-y\|^{2}\right)}$

where $\|\not\|$ denote the two norms.

Theorem 1. The distance function in Equation 6 is a metric (Zhang and Chen, 2004).

Theorem 1 shows that $\operatorname{dist}(x, y)$ satisfies the following three conditions:

(A) $\operatorname{dist}(x, y)>0, \forall x \neq y, \operatorname{dist}(x, x)=0$, 
(B) $\operatorname{dist}(x, y)=\operatorname{dist}(y, x)$,

(C) $\operatorname{dist}(x, y) \leq \operatorname{dist}(x, z)+\operatorname{dist}(z, y) \quad \forall z$.

The advantage of the new distance function is that the value of it will not be more than one regardless of the difference of $x$ and $y$ whether large or small. In other words, $\operatorname{dist}(x, y) \in[0,1)$. If we use Euclidean distance $\operatorname{dist}(x, y)=\|x-y\|$, its range will be from 0 to infinity. In the model of Soyster (1973), the "protection" of constraint will be large if the Euclidean distance is large, so the feasible region of the model will change a little and the model will be very conservatism. In order to reduce the conservatism, we used the new distance function to set up the new robust counterpart.

We motivate the new formulation by considering the th constraint of the nominal problem $a_{i}^{T} x \leq b_{i}$. Let $J_{i}$ be the set of coefficients $a_{i j}, j \in J_{i}$ that are subject to parameter uncertainty; $\tilde{a}_{i j}, j \in J_{i}$ takes values according to a symmetric distribution with mean equal to the nominal value $a_{i j}$ in the interval $\left[a_{i j}-\widehat{a}_{i j}, a_{i j}+\widehat{a}_{i j}\right]$. For every $i$, we introduce a parameter $\beta_{i}$, the role of it is to adjust the robustness of the proposed method against the level of conservatism of the solution. We consider the following formulation:

$\max c^{T} x$

$$
\begin{array}{ll}
\text { s.t. } & \left.\sum_{j} a_{i j} x_{j}+\beta \sum_{j \in J_{i}} \sqrt{1-\exp \left(-\left\|\tilde{a}_{i j}-a_{i j}\right\|^{2}\right.}\right) y_{j} \leq b_{i} \quad \forall i \\
& -y_{j} \leq x_{j} \leq y_{j} \quad \forall j \in J_{i} \\
& l \leq x \leq u \\
& y \geq 0
\end{array}
$$

If $\beta_{i}=0$ or $\tilde{a}_{i j}=a_{i j}$, the constrains are equivalent to that of the nominal problem. We can see that the model (7) is also a linear optimization.

\section{PROBABILITY BOUNDS OF CONSTRAINT VIOLATION}

Here, we show that under the model of data uncertainty $U$, the robust solution is feasible with high probability. Where $U=\left[a_{i j}-\hat{a}_{i j}, a_{i j}+\hat{a}_{i j}\right]$ denote the uncertainty set. The role of the parameter $\beta_{i}$ is to adjust the robustness of the method against the level of conservatism of the solution.

\section{Proposition 1}

Let $x^{*}$ be an optimal solution of Equation 7. Suppose that the data in matrix $A$ are subjected to the model of data uncertainty $U$. Then, the probability that the ith constraint is violated satisfies:

$\operatorname{Pr}\left(\sum_{j} \tilde{a}_{i j} x_{j}^{*}>b_{i}\right) \leq \operatorname{Pr}\left(\sum_{j \in J_{i}} \mu_{i j} \eta_{i j} \geq \beta_{i}\right)$ $\mu_{i j}=\frac{\hat{a}_{i j} x_{j}^{*}}{\sqrt{1-\exp \left(-\left\|\tilde{a}_{i j}-a_{i j}\right\|^{2}\right)}\left|x_{j}^{*}\right|}$

$$
=\frac{\hat{a}_{i j} x_{j}^{*}}{\sqrt{1-\exp \left(-\hat{a}_{i j}^{2}\right) \mid x_{j}^{*}}} \quad \forall j \in J_{i} \text {. }
$$

Proof: Let $x^{*}$ be an optimal solution of Equation 7. Then, the probability of violation of the th constraint is as follows:

$$
\begin{aligned}
& \operatorname{Pr}\left(\sum_{j} \tilde{a}_{i j} x_{j}^{*}>b_{i}\right)=\operatorname{Pr}\left(\sum_{j \notin J_{i}} a_{i j} x_{j}^{*}+\sum_{j \in J_{i}} \tilde{a}_{i j} x_{j}^{*}>b_{i}\right) \\
& \leq \operatorname{Pr}\left(\sum_{j \notin J_{i}} a_{i j} x_{j}^{*}+\sum_{j \in J_{i}} \tilde{a}_{i j} x_{j}^{*}>\sum_{j \notin J_{i}} a_{i j} x_{j}^{*}\right. \\
& \left.\left.+\sum_{j \in J_{i}} a_{i j} x_{j}^{*}+\beta_{i} \sum_{j \in J_{i}} \sqrt{1-\exp \left(-\left\|\tilde{a}_{i j}-a_{i j}\right\|^{2}\right.}\right)\left|x_{j}^{*}\right|\right) \\
& =\operatorname{Pr}\left(\sum_{j \in J_{i}} \tilde{a}_{i j} x_{j}^{*}>\sum_{j \in J_{i}} a_{i j} x_{j}^{*}+\beta_{i} \sum_{j \in J_{i}} \sqrt{1-\exp \left(-\left\|\tilde{a}_{i j}-a_{i j}\right\|^{2}\right)}\left|x_{j}^{*}\right|\right) \\
& =\operatorname{Pr}\left(\sum_{j \in J_{i}} \hat{a}_{i j} x_{j}^{*} \eta_{i j}>\beta_{i} \sum_{j \in J_{i}} \sqrt{1-\exp \left(-\left\|\tilde{a}_{i j}-a_{i j}\right\|^{2}\right)}\left|x_{j}^{*}\right|\right) \\
& =\operatorname{Pr}\left(\sum_{j \in J_{i}} \frac{a_{i j} x_{j}^{*}}{\left.\left.\sqrt{1-\exp \left(-\left\|\tilde{a}_{i j}-a_{i j}\right\|^{2}\right.}\right) \mid x_{j}^{*} \eta_{i j}>\beta_{i}\right)}\right. \\
& =\operatorname{Pr}\left(\sum_{j \in J_{i}} \mu_{i j} \eta_{i j}>\beta_{i}\right) \leq \operatorname{Pr}\left(\sum_{j \in J_{i}} \mu_{i j} \eta_{i j} \geq \beta_{i}\right)
\end{aligned}
$$

Inequality Equation 8 follows from Equation 7 , since $x^{*}$ satisfies

$$
\sum_{j} a_{i j} x_{j}+\beta_{i} \sum_{j \in J_{i}} \sqrt{1-\exp \left(-\left\|\tilde{a}_{i j}-a_{i j}\right\|^{2}\right)} y_{j} \leq b_{i}
$$

The next naturally provides a bound that is independent of the solution $x^{*}$.

Theorem 2: If $\eta_{i j}, j \in J_{i}$ are independent and symmetrically distributed random variables in $[-1,1]$, then

$$
\operatorname{Pr}\left(\sum_{j \in J_{i}} \mu_{i j} \eta_{i j} \geq \beta_{i}\right) \leq \exp \left(-\frac{\beta_{i}^{2}}{2 \mu_{i}^{*}\left|J_{i}\right|}\right)
$$

where

$\mu_{i}^{*}=\max \left\{\mu_{i j}^{2} \mid j \in J_{i}\right\}=\max \left\{\frac{\hat{a}_{i j}^{2}}{1-\exp \left(-\hat{a}_{i j}{ }^{2}\right)} \mid j \in J_{i}\right\}$.

Proof: Let $\theta>0$. Then, 
$\operatorname{Pr}\left(\sum_{j \in J_{i}} \mu_{i j} \eta_{i j} \geq \beta_{i}\right) \leq \frac{E\left[\exp \left(\theta \sum_{j \in J_{i}} \mu_{i j} \eta_{i j}\right)\right]}{\exp \left(\theta \beta_{i}\right)}$

$=\frac{\prod_{j \in J_{i}} E\left[\exp \left(\theta \mu_{i j} \eta_{i j}\right)\right]}{\exp \left(\theta \beta_{i}\right)}$

$=\frac{\Pi_{j \in J_{i}} 2 \int_{0}^{1} \sum_{k=0}^{\infty}\left(\left(\theta \mu_{i j} \eta\right)^{2 k} /(2 k) !\right) d F_{\eta_{i j}}(\eta)}{\exp \left(\theta \beta_{i}\right)}$

$\leq \frac{\prod_{j \in J_{i}} \sum_{k=0}^{\infty}\left(\left(\theta \mu_{i j}\right)^{2 k}\right) /(2 k) !}{\exp \left(\theta \beta_{i}\right)}$

$\leq \frac{\Pi_{j \in J_{i}} \exp \left(\theta^{2} \mu_{i j}^{2} / 2\right)}{\exp \left(\theta \beta_{i}\right)}$

$\leq \exp \left(\left|J_{i}\right| \mu_{i}^{*} \frac{\theta^{2}}{2}-\theta \beta_{i}\right)$

$=\exp \left(-\frac{\beta_{i}^{2}}{2 \mu_{i}^{*}\left|J_{i}\right|}\right) \exp \left[\left(\theta \sqrt{\frac{\mu_{i}^{*}\left|J_{i}\right|}{2}}-\frac{\beta_{i}}{\sqrt{2 \mu_{i}^{*}\left|J_{i}\right|}}\right)^{2}\right]$

$\Rightarrow \operatorname{Pr}\left(\sum_{j \in J_{i}} \mu_{i j} \eta_{i j} \geq \beta_{i}\right)$

$\leq \exp \left(-\frac{\beta_{i}^{2}}{2 \mu_{i}^{*}\left|J_{i}\right|}\right) \min _{\theta>0}\left\{\exp \left[\left(\theta \sqrt{\frac{\mu_{i}^{*}\left|J_{i}\right|}{2}}\right.\right.\right.$

$\left.\left.\left.-\frac{\beta_{i}}{\sqrt{2 \mu_{i}^{*}\left|J_{i}\right|}}\right)^{2}\right]\right\}$

$=\exp \left(-\frac{\beta_{i}^{2}}{2 \mu_{i}^{*}\left|J_{i}\right|}\right)$

Inequality Equation 9 follows from Markov's inequality (Bojanov, 1982). Equations 10 and 11 follow from the independence and symmetric distribution assumption of the random variables $\eta_{i j}$.

\section{EXPERIMENTAL RESULTS}

Here, we applied our method to the problem AFIRO and ADLITTLE from the Net Lib library of problems. The problem AFIRO is a linear optimization problem with 28 rows, 32 columns and optimum objective value 464.7531. It contains coefficients such as 1.399999999999999, $\quad 0.9599999999999999$, $0.8599999999999999,0.4299999999999999$ and so on, which seem unnecessarily precise. In our study, we assume that the uncertain data is only included in the row of [X44]. In other words, the uncertain data is only included in the constraint of $-x_{23}+1.399999999999999 x_{36} \leq 0$. In order to verify the robustness of the model, we take the maximum range of disturbance from 0.2 to 2.0. We use LINGO 10.0 on a Pentium II 400 PC to solve this problem.

The problem ADLITTLE is a linear optimization problem with 57 rows, 97 columns and optimum objective value 225495.0. It contains coefficients such as 39.79999999999999, 43.39999999999999, $0.1199999999999999 \mathrm{E}-02,0.1809999999999999$ and so on, which seem unnecessarily precise. In our study, we assume that the uncertain data is only included in the row of 27. In other words, the uncertain data is only included in the constraint of $0.679 \cdot x 160+0.679 \cdot x 161+0.534 \cdot x 176+0.534 \cdot x 177 \leq 290$ and the coefficients of the variables $x 161, x 176$, $x 177$ have disturbances. In order to verify the robustness of the model, we take the maximum range of disturbance from 0.2 to 2.0. We use LINGO10.0 on a Pentium II 400 PC to solve this problem.

Tables 1 and 3 show the comparison of different models under different disturbance values when $\Gamma_{i}=\beta_{i}=0.5$. We can see that comparison with the model of Soyster (1973) and Bertsimas and Sim (2004), our approach in ensuring the robustness of solution, but it did not lose a lot of optimality. We can see that the new model obtained the optimal value under the influence of the uncertain parameters which have a smaller rate of change. Thus, the new model reduces the conservatism.

Tables 2 and 4 show the comparison of different models under different adjustment factors when $\widehat{a}_{23}=\widehat{a}_{36}=0.6$. We can see that the rate of optimal value change of our model is only half of the model of Bertsimas and Sim (2004) under the same conditions of the uncertain data and the adjust parameters. Thus, our model has good optimality while maintaining the robustness of solution.

\section{Conclusion}

In this paper, a new robust counterpart is proposed for linear optimization with uncertain data. Our method provided solutions that ensure probabilistic guarantees for those constraints that will be satisfied as data change, and the probability bounds of constraint violation do not depend on the robust model. Our robust counterpart is also a linear optimization problem, which will make our approach easy to solve. 
Table 1. Comparison of different models under different disturbance values $\left(\Gamma_{i}=\beta_{i}=0.5\right)$.

\begin{tabular}{|c|c|c|c|c|c|c|}
\hline \multirow{2}{*}{$\widehat{a}_{23}=\widehat{a}_{36}=$} & \multicolumn{2}{|c|}{ Soyster model of Equation 2} & \multicolumn{2}{|c|}{ Bertsimas and Sim model of Equation 5} & \multicolumn{2}{|c|}{ New model of Equation 7} \\
\hline & Optimal value & $\%$ change & Optimal value & $\%$ change & Optimal value & $\%$ change \\
\hline 0.2 & -415.8014 & 10.5328 & -448.4359 & 3.5109 & -464.5382 & 0.0462 \\
\hline 0.4 & -377.7278 & 18.7251 & -432.1186 & 7.0219 & -461.7192 & 0.6528 \\
\hline 0.6 & -347.2689 & 25.2788 & -415.8014 & 10.5328 & -452.3739 & 2.6636 \\
\hline 0.8 & -322.3480 & 30.6410 & -399.4841 & 14.0438 & -435.8104 & 6.2275 \\
\hline 1.0 & -301.5806 & 35.1095 & -383.1669 & 17.5547 & -415.8467 & 10.5231 \\
\hline 1.2 & -48.63589 & 89.5351 & -366.8496 & 21.0657 & -397.3345 & 14.5063 \\
\hline 1.4 & -48.63589 & 89.5351 & -350.5323 & 24.5767 & -383.0545 & 17.5789 \\
\hline 1.6 & -48.63589 & 89.5351 & -334.2151 & 28.0876 & -373.4429 & 19.6470 \\
\hline 1.8 & -48.63589 & 89.5351 & -317.8978 & 31.5986 & -367.6485 & 20.8938 \\
\hline 2.0 & -48.63589 & 89.5351 & -301.5806 & 35.1095 & -364.4795 & 21.5757 \\
\hline
\end{tabular}

Table 2. Comparison of different models under different adjustment factors ( $\hat{a}_{23}=\widehat{a}_{36}=0.6$ ).

\begin{tabular}{c|cc|cc}
\hline \multirow{2}{*}{$\Gamma_{i}=\beta_{i}=$} & \multicolumn{2}{c|}{ Bertsimas and Sim model of Equation 5 } & \multicolumn{2}{c}{ New model of Equation 7 } \\
\hline 0.2 & Optimal value & \% change & Optimal value & \% change \\
\hline 0.4 & -445.1724 & 4.2131 & -459.7057 & 1.0860 \\
0.6 & -425.5917 & 8.4263 & -454.7867 & 2.1445 \\
0.8 & -406.0110 & 12.6394 & -449.9914 & 3.1762 \\
1.0 & -386.4303 & 16.8526 & -445.3150 & 4.1825 \\
1.2 & -366.8496 & 21.0657 & -440.7533 & 5.1640 \\
1.4 & -361.6968 & 22.1744 & -436.3020 & 6.1218 \\
1.6 & -357.2980 & 23.1209 & -431.9573 & 7.0566 \\
1.8 & -353.4991 & 23.9383 & -427.7153 & 7.9693 \\
2.0 & -350.1852 & 24.6513 & -423.5724 & 8.8608 \\
& -347.2689 & 25.2788 & -419.5252 & 9.7316 \\
\hline
\end{tabular}

Table 3. Comparison of different models under different disturbance values $\left(\Gamma_{i}=\beta_{i}=0.5\right)$.

\begin{tabular}{c|cc|cc}
\hline$\hat{a}_{161}=\hat{a}_{176}$ & \multicolumn{2}{c|}{ Bertsimas and Sim model of Equation 5 } & \multicolumn{2}{c}{ New model of Equation 7 } \\
\cline { 2 - 5 }$=\hat{a}_{177}=$ & Optimal value & \% change & Optimal value & \% change \\
\hline 0.0001 & 225495.5 & $2.2173 e-004$ & 225495.0 & 0 \\
0.001 & 225500.0 & 0.0022 & 225495.0 & 0 \\
0.01 & 225545.5 & 0.0224 & 225495.0 & 0 \\
0.1 & 226021.8 & 0.2336 & 225495.8 & $3.5478 \mathrm{e}-004$ \\
0.3 & 227332.3 & 0.8148 & 225557.0 & 0.0275 \\
0.5 & 244221.4 & 8.3046 & 225904.8 & 0.1817 \\
0.7 & 259360.5 & 15.0183 & 226751.8 & 0.5574 \\
0.9 & 273173.2 & 21.1438 & 230356.5 & 2.1559 \\
\hline
\end{tabular}

\section{ACKNOWLEDGEMENTS}

This work is supported by the National Natural Science
Foundation of China (Grant No.60974082), and the Shaanxi Province (Nos.09JK722 and 11JK1051). Science Plan Foundation of the Education Bureau of 
Table 4. Comparison of different models under different adjustment factors ( $\left.\widehat{a}_{161}=\widehat{a}_{176}=\widehat{a}_{177}=0.6\right)$.

\begin{tabular}{c|cc|cc}
\hline \multirow{2}{*}{$\Gamma_{i}=\beta_{i}=$} & \multicolumn{2}{|c|}{ Bertsimas and Sim model of Equation $\mathbf{5}$} & \multicolumn{2}{c}{ New model of Equation 7 } \\
\cline { 2 - 5 } & Optimal value & \% change & Optimal value & \% change \\
\hline 0.2 & 226863.1 & 0.6067 & 225801.2 & 0.1358 \\
0.4 & 242671.7 & 7.6173 & 226107.4 & 0.2716 \\
0.6 & 260820.5 & 15.6658 & 226413.6 & 0.4074 \\
0.8 & 277016.9 & 22.8484 & 226719.8 & 0.5432 \\
1.0 & 291121.3 & 29.1032 & 227110.9 & 0.7166 \\
1.5 & 319379.2 & 41.6347 & 229081.4 & 1.5905 \\
\hline
\end{tabular}

\section{REFERENCES}

Ben-Tal A, Nemirovski A (1999). Robust solutions of uncertain linear programs. Oper. Res. Lett., 25(1): 1-13.

Ben-Tal A, Nemirovski A (2000). Robust solutions of Linear Programming problems contaminated with uncertain data. Math. Progr., 88(3): 411-424.

Ben-Tal A, Goryashko A, Guslitzer E, Nemirovski A (2004). Adjustable robust solutions of uncertain linear programs. Math. Progr., 99(2): 351-376.

Bertsimas D, Brown D (2009). Constructing Uncertainty Sets for Robust Linear Optimization. Oper. Res., 57(6): 1483-1495.

Bertsimas D, Pachamanova D, Sim M (2004). Robust linear optimization under general norms. Oper. Res. Lett., 32(6): 510-516.

Bertsimas D, Sim M (2004). The price of Robustness. Oper. Res., 52(1): 35-53.

Bertsimas D, Sim M (2006). Tractable Approximations to robust conic optimization problems. Math. Progr. Ser., B 107(1-2): 5-36.

Bojanov BD (1982). An extension of the Markov inequality. J. Approx. Theory, (35):181-190.
Chen X, Zhang YH (2009). Uncertain Linear Programs: Extended Affinely Adjustable Robust Counterparts. Oper. Res., 57(6): 14691482.

Ghaoui LEI, Lebret H (1997). Robust solutions to least-squares problems with uncertain data. SIAM J. Matrix Anal. Appl., 18(4): 1035-1064.

Ghaoui LEI, Oustry F, Lebret H (1998). Robust solutions to uncertain semidenite programs. SIAM J. Optim., 9(1): 33-52.

Soyster AL (1973). Convex Programming with Set-Inclusive Constraints and Applications to Inexact Linear Programming. Oper. Res., 21(5): 1154-1157.

Wu KL, Yang MS (2002). Alternative c-means clustering algorithms. Patt. Recognit., 35(10): 2267-2278.

Zhang DQ, Chen SC (2004). A comment on "Alternative c-means clustering algorithms." Patt. Recognit., (37): 173-174. 